\title{
Weight change and sulfonylurea therapy are related to 3 year change in microvascular function in people with type 2 diabetes
}

\author{
Francesco Casanova ${ }^{1} \cdot \operatorname{Kim}$ M. Gooding ${ }^{1} \cdot$ Angela C. Shore $^{1} \cdot$ Damilola D. Adingupu ${ }^{1} \cdot$ David Mawson $^{1,2} \cdot$ Claire Ball $^{2}$. \\ Christine Anning ${ }^{2} \cdot$ Kunihiko Aizawa $^{1} \cdot$ Philip E. Gates ${ }^{1} \cdot$ W. David Strain ${ }^{1}$ (D)
}

Received: 2 September 2019 / Accepted: 17 February 2020 / Published online: 17 March 2020

(C) The Author(s) 2020

\begin{abstract}
Aims/hypothesis Although cardiovascular disease is the biggest cause of death in people with diabetes, microvascular complications have a significant impact on quality of life and financial burden of the disease. Little is known about the progression of microvascular dysfunction in the early stages of type 2 diabetes before the occurrence of clinically apparent complications. We aimed to explore the determinants of endothelial-dependent and -independent microvascular function progression over a 3 year period, in people with and without both diabetes and few clinical microvascular complications.

Methods Demographics were collected in 154 participants with type 2 diabetes and in a further 99 participants without type 2 diabetes. Skin microvascular endothelium-dependent response to iontophoresis of acetylcholine and endotheliumindependent responses to sodium nitroprusside were measured using laser Doppler fluximetry. All assessments were repeated 3 years later.

Results People with type 2 diabetes had impaired endothelial-dependent microvascular response compared with those without (AUC 93.9 [95\% CI 88.1, 99.4] vs $111.9[102.3,121.4]$ arbitrary units [AU] $\times \min , p<0.001$, for those with vs without diabetes, respectively). Similarly, endothelial-independent responses were attenuated in those with diabetes $(63.2[59.2,67.2]$ vs 75.1 $[67.8,82.4] \mathrm{AU} \times \min$, respectively, $p=0.002)$. Mean microvascular function declined over 3 years in both groups to a similar degree ( $p_{\text {interaction }} 0.74$ for response to acetylcholine and 0.69 for response to sodium nitroprusside). In those with diabetes, use of sulfonylurea was associated with greater decline ( $p=0.022$ after adjustment for co-prescriptions, change in $\mathrm{HbA}_{1 \mathrm{c}}$ and weight), whereas improving glycaemic control was associated with less decline of endothelial-dependent microvascular function $(p=$ 0.03). Otherwise, the determinants of microvascular decline were similar in those with and without diabetes. The principal determinant of change in microvascular function in the whole population was weight change over 3 years, such that those that lost $\geq 5 \%$ weight had very little decline in either endothelial-dependent or -independent function compared with those that were weight stable, whereas those who gained weight had a greater decline in function (change in endothelial-dependent function was $1.2[95 \% \mathrm{CI}-13.2,15.7] \mathrm{AU} \times \min$ in those who lost weight; $-15.8[-10.5,-21.0] \mathrm{AU} \times \min$ in those with stable weight; and $-37.8[-19.4,-56.2] \mathrm{AU} \times \min$ in those with weight gain; $\left.p_{\text {trend }}<0.001\right)$. This association of weight change with change in endothelial function was driven by people with diabetes; in people without diabetes, the relationship was nonsignificant.

Conclusions/interpretation Over 3 years, physiological change in weight was the greatest predictor of change in microvascular function.
\end{abstract}

Keywords Diabetes $\cdot$ Epidemiology $\cdot$ Microvascular $\cdot$ Population $\cdot$ Weight loss

Electronic supplementary material The online version of this article (https://doi.org/10.1007/s00125-020-05125-4) contains peer-reviewed but unedited supplementary material, which is available to authorised users.

\section{W. David Strain}

d.strain@exeter.ac.uk

1 Diabetes and Vascular Medicine Research Centre, Institute of Biomedical and Clinical Science and University of Exeter College of Medicine and Health, Royal Devon \& Exeter Hospital, Barrack Road, Exeter EX2 5AX, UK
2 NIHR Exeter Clinical Research Facility, Royal Devon \& Exeter NHS Foundation Trust and University of Exeter, College of Medicine and Health, Exeter, UK 


\section{Research in context}

\section{What is already known about this subject?}

- Although cardiovascular disease is the biggest cause of death in people with diabetes, microvascular complications are the most feared and significantly impact on quality of life

- Once microvascular complications are established, good glycaemic control has been demonstrated to reduce their progression

- Little is known about the predictors of microvascular decline before clinically apparent complications are present

What is the key question?

- What are the predictors of microvascular endothelium-dependent and -independent functional decline over 3 years in people without, or with only very early evidence of, microvascular complications of diabetes?

What are the new findings?

- Improved glycaemic control was associated with less decline in endothelium-dependent microvascular function, independent of baseline $\mathrm{HbA}_{1 \mathrm{c}}$

- The use of sulfonylurea therapy was associated with greater microvascular decline, independent of the effect on $\mathrm{HbA}_{1 \mathrm{c}}$ and weight

- Weight change over 3 years was the best predictor of changes in endothelium-dependent microvascular function, with a $5 \%$ weight loss associated with arresting the decline in function, and a $5 \%$ weight gain doubling the rate of decline

How might this impact on clinical practice in the foreseeable future?

- When choosing therapies in early diabetes, the impact on weight should be considered in addition to blood glucose

\begin{tabular}{|c|c|}
\hline \multicolumn{2}{|c|}{ Abbreviations } \\
\hline $\mathrm{ACh}$ & Acetylcholine \\
\hline $\mathrm{AU} \times \min$ & $\begin{array}{l}\text { Arbitrary units } \times \text { min (units for AUC of the } \\
\text { response curve) }\end{array}$ \\
\hline CVD & Cardiovascular disease \\
\hline LDI & Laser Doppler imager \\
\hline NHS & National Health Service \\
\hline SNP & Sodium nitroprusside \\
\hline SUMMIT & $\begin{array}{l}\text { SUrrogate markers for Micro- and Macro- } \\
\text { vascular hard endpoints for Innovative diabetes } \\
\text { Tools }\end{array}$ \\
\hline
\end{tabular}

\section{Introduction}

In recent years there has been considerable focus on the impact of type 2 diabetes on cardiovascular disease (CVD) as the principal cause of death in people with diabetes. Microvascular complications of diabetes, however, have a major impact on the quality of life of people with diabetes and are the most feared in people with diabetes [1]. Further, both structural and functional changes in the microvasculature significantly alter the peripheral resistance and thus haemodynamic stress on the heart and other organs. It is thought that this is how they play an independent part in the initiation and progression of atherosclerotic plaques [2].

Whereas the pathophysiological mechanisms of large artery disease have been well studied, the physiology, mechanisms and functional changes over time at the microvascular level are poorly understood. It is known that determinants of microvascular disease progression differ from those at the macrovascular level in type 2 diabetes and thus findings in the large vessels cannot be extrapolated to small vessels [3]. The principal determinants of microvascular disease progression appear to be hyperglycaemia, and resultant advanced glycaemic endproducts (AGEs) and hyperinsulinaemia in those with diabetes [4]. Eutrophic (inward) remodelling within the microcirculation in response to hypertension reduces the flow through the vessel, thereby increasing the resistance (itself a driver for further hypertension), and limits potential for endothelially mediated relaxation of the vessels [5]. Conversely, hypertrophic (outward) remodelling maintains the flow, but limits the diffusion capacity of the vessels. The respective causes of these alterations are poorly understood; however, both result in attenuated myogenic responses to sheer stress [6]. The clinically measurable impact is an increased urinary AER. It is 
important to note that the urinary albumin is not itself a disease progression determinant, but rather a measure of systemic microvascular dysfunction.

Urinary AER, often regarded as the quantifiable hallmark of diabetic microvascular disease, represents the presence of substantial microvascular impairment $[7,8]$. The natural history of preclinical changes is poorly understood because of the paucity of long-term studies in relevant surrogates. The techniques used to explore preclinical microvascular dysfunction have developed substantially in recent times [9]. The regulatory mechanisms for the skin microcirculation appear to be different from forearm blood flow [10], and responses in these two vascular territories do not normally correlate in healthy individuals [11, 12]. The use of laser Doppler fluximetry with iontophoresis of acetylcholine (ACh) and sodium nitroprusside (SNP) enables mechanistic studies to non-invasively elucidate endothelialdependent and -independent effects [13, 14]. Skin microvascular responses have been demonstrated to be reduced in people with type 2 diabetes [7], and associated with left ventricular hypertrophy [15], urinary AER [16], retinopathy [17], coronary artery disease [18] and symptomatic angina independent of atherosclerosis [19]. A recent systematic review and network analysis found progressive microvascular impairment across the spectrum of metabolic health, from a healthy population to those with obesity, glucose impairment and diabetes mellitus [20].

We aimed to explore the determinants of endothelialdependent and -independent microvascular function progression over a 3 year period, in people both with and without diabetes and few clinical microvascular complications.

\section{Methods}

This study conformed to the Declaration of Helsinki and was approved by the National Research Ethics Service Southwest (10/H0206/67). All participants gave informed written consent.

Study population Participants were adult men and women, enhanced with individuals with type 2 diabetes and/or proven CVD recruited as part of the 'SUrrogate markers for Microand Macro-vascular hard endpoints for Innovative diabetes Tools' (SUMMIT) programme (Innovative Medicines Initiative [IMI] grant number 115006; http://www.imisummit.eu) via the Peninsula Research Bank, part of the National Institute for Health Research (NIHR) Exeter Clinical Research Facility, and the Royal Devon and Exeter National Health Service (NHS) clinical service.

Type 2 diabetes was diagnosed according to current guidelines by the patients' physicians based on an $\mathrm{HbA}_{1 \mathrm{c}}$ above $48 \mathrm{mmol} / \mathrm{mol}(6.5 \%)$. Patients diagnosed with type 2 diabetes under the age of 35 or treated with insulin within 12 months of diagnosis were not included in the study. CVD was defined as previously described [7], and this included a medical history of myocardial infarction, percutaneous coronary intervention, coronary arterial bypass graft, unstable angina and specialistdiagnosed cerebrovascular events from stroke and cardiology units and clinics at the Royal Devon and Exeter Hospital, UK. Exclusion criteria included any malignancy requiring active treatment, any treated chronic inflammatory disease, renal replacement therapy and end-stage renal disease.

Study visits All participants had multiple visits for both baseline and follow-up assessments 3 years apart (mean follow-up time: $3.14 \pm 0.21$ years). For both baseline and follow-up, the microvascular studies were organised at the same time of day or as close to this as possible.

All participants refrained from eating and drinking (except water) for at least $2 \mathrm{~h}$ before the visit, and avoided smoking, caffeine, alcohol and strenuous exercise on the study day. Medications were omitted on the morning of the study wherever possible. All vasoactive drugs were withheld for a minimum of $12 \mathrm{~h}$ prior to the studies. Participants followed the same protocol for medications at both visits, allowing their results to be compared. All studies were performed in temperature-controlled laboratories $\left(23 \pm 1^{\circ} \mathrm{C}\right)$, with the participants lying in the supine position following an acclimatisation period of at least $20 \mathrm{~min}$.

Screening and blood tests For both baseline and follow-up appointments, screening assessment included anthropometry, electrocardiogram and an interview that included medical history and pharmacotherapy. For anthropometry, height, weight and waist/hip ratio were measured using a standard protocol. Brachial blood pressure was measured in the supine position using an automated blood pressure device (Omron M6; Omron Healthcare Europe, Hoofddorp, the Netherlands) and the mean of three measurements was used for analysis.

All blood tests were measured from a $10 \mathrm{~h}$ fasting blood sample collected on the day of the study or within a week of the study day when not possible. Participants' fasting glucose and lipid concentrations were measured by the hospital's pathology service (Exeter Pathology Services, Royal Devon and Exeter NHS Foundation Trust), in accordance with the UK National Quality Assessment Scheme. Urinary AER was measured using a timed overnight urine collection protocol and AER was calculated as:

$$
\begin{array}{r}
\text { albumin }(\mathrm{mg} / \mathrm{dl}) \times \text { volume of urine }(\mathrm{dl}) \times 1000] \\
/ \text { time of urine collection }(\mathrm{min})
\end{array}
$$

Albumin was measured by the hospital's pathology service (Exeter Pathology Services, Royal Devon and Exeter NHS Foundation Trust) in accordance with the UK National Quality Assessment Scheme. Albumin/creatinine ratio was 
measured from a random spot urine sample taken on the day of one of the visits. Analyses of albumin and creatinine concentrations were performed using immunoturbidimetric and Jaffe methods, respectively, with a detection limit for albumin of $3.0 \mathrm{mg} / \mathrm{l}$. Diabetic retinopathy was graded from two-field photography (nasal and macular view) using the English National Diabetic Retinopathy Grading scheme; for the purposes of this study, diabetic retinopathy was grouped into the following categories: no diabetic retinopathy; nonproliferative diabetic retinopathy; proliferative diabetic retinopathy; and previous laser treatment for proliferative diabetic retinopathy and/or clinically significant macular oedema. Neuropathy was assessed with a vibration perception test on the pulp of the hallux of left and right toes using a neurothesiometer (SLS, Nottingham, UK). A threshold of $25 \mathrm{~V}$ was used to define neuropathy.

Skin microvascular assessments: Iontophoresis of endothelialdependent (acetylcholine) and -independent (sodium nitroprusside) vasodilators Response to pharmacological stimulation of the skin blood vessels was conducted as previously described [7]. A solid-state laser Doppler imager (LDI) (LDI2; Moor Instruments, Axminster, UK) was used to measure skin perfusion, with the head of the LDI positioned $50 \mathrm{~cm}$ above the skin. The LDI was set to scan a region of $4.8 \mathrm{~cm}^{2}$. The LDI was interfaced with a computer equipped with moorLDI software (Research Version 5.3). A batterypowered iontophoresis controller (MIC 1; Moor Instruments) was used to provide the current for iontophoresis.

After very gentle skin cleaning with alcohol wipes, Perspex direct electrode chambers were attached to the volar aspect of the forearm using a double-sided adhesive ring, avoiding visible veins, freckles and hair. Chambers were filled with solutions under investigation; ACh (1\% Miochol-E dissolved in mannitol; Novartis, Camberly, UK) and SNP (0.25\%) $(25 \mathrm{mg} / \mathrm{ml}$ Nitropress dissolved in $0.45 \%$ saline [ $77 \mathrm{mmol} / 1$ $\mathrm{NaCl}$; ; Hospira, Lake Forest, IL, USA). A glass cover slip was placed on the drug chamber to prevent reflection artefacts from light scattered by the convex surface of the solution in the chamber. An indifferent electrode was attached to the volar aspect of the participant's wrist and connected to the chamber terminal to complete the circuit.

$\mathrm{ACh}$ was delivered using five pulses $(100 \mu \mathrm{A}$ each) of anodal current with a $60 \mathrm{~s}$ interval between each dose (total charge $12 \mathrm{mC}$ ). Perfusion was assessed at rest and then every $20 \mathrm{~s}$ for $6 \mathrm{~min}$ from the start of the charge period, with no interval between scans.

SNP was delivered by cathodal current. A single pulse of $200 \mu \mathrm{A}$ was introduced for $60 \mathrm{~s}$ (total charge $10 \mathrm{mC}$ ). Forearm skin erythrocyte flux was again recorded at rest and then every $20 \mathrm{~s}$ for $6 \mathrm{~min}$ from the start of the charge period.

Median perfusion response was calculated for each image obtained by the LDI using the total area to which the drug was applied. Skin microvascular perfusion was measured as the area under the response curve, normalised to resting flow, using the trapezius rule for both $\mathrm{ACh}$ and SNP and expressed in arbitrary units $\times \min (\mathrm{AU} \times \min )$.

Statistical analysis Analysis was performed on continuous data to maximise power. Normality was formally assessed in variables of interest using the Kolmogorov-Smirnov test against theoretical distribution. Skewed variables were appropriately transformed, and medians [IQRs] are presented. Statistical significance for categorical variables was calculated using the $\chi^{2}$ test, and Student's $t$ tests or one-way ANOVA for continuous variables, at baseline and follow-up. Where no appropriate transformation was available, nonparametric alternatives (Mann-Whitney $U$ test) were applied and median $[\mathrm{IQR}]$ is presented. Differences in change over time between groups were analysed using a two-way ANOVA (time $\times$ group) and $p_{\text {interaction }}$ is reported. Multiple regression was used to investigate the predictors of change over time. In exploring mechanisms for the effect of time, potential confounding effects of age and sex, and mechanistic factors, were considered. Mechanistic factors known to affect microvascular function included blood pressure indices (brachial systolic, brachial diastolic and mean arterial pressure), body size (weight, BMI, waist circumference and waist/hip ratio), insulin resistance (fasting glucose and $\mathrm{HbA}_{1 \mathrm{c}}$ ) and lipid profile (total cholesterol, LDL-cholesterol, HDL and triacylglycerols). A single model was developed by choosing a variable from each of these groups, based on the greatest increase in the amount of variance explained by their inclusion in the bivariate model against AUC for endothelial-dependent response to ACh. Additional analyses were performed assessing the impact of treatment with agents thought to impact vascular function, including statins, drugs acting on the renin angiotensin system and glucose-lowering drugs. All results of multivariate modelling are presented after adjustment for sex and age at baseline. When considering the principle variables of interest in the microcirculation, a result was deemed significant if $p \leq 0.05$. When comparing other variables between groups, $p$ values should be regarded as indicative.

\section{Results}

Baseline characteristics of the cohort recruited for this study are presented in Table 1. Due to the demographics of the local population, all participants were of white European descent. All of the female participants were postmenopausal at recruitment to the baseline visit. Those with type 2 diabetes were slightly older and had higher $\mathrm{BMI}$ and $\mathrm{HbA}_{1 \mathrm{c}}$, but a more favourable lipid profile, than those without diabetes, likely representing more frequent statin prescription. Blood pressure was similar in those with and without diabetes, although 
Table 1 Baseline characteristics of the cohort stratified by the presence of diabetes (DM) or absence of diabetes (No DM)

\begin{tabular}{|c|c|c|c|}
\hline Characteristic & No DM $(n=99)$ & $\mathrm{DM}(n=154)$ & $p$ \\
\hline Sex (male/female) $(n)$ & $41 / 58$ & $42 / 112$ & 0.019 \\
\hline Age (years) & $64.8(63.0,66.5)$ & $67.9(66.6,69.2)$ & 0.004 \\
\hline Weight (kg) & $75.6(72.9,78.3)$ & $91.2(88.6,93.8)$ & $<0.001$ \\
\hline Height (m) & $1.70(1.68,1.72)$ & $1.72(1.71,1.73)$ & 0.145 \\
\hline BMI $\left(\mathrm{kg} / \mathrm{m}^{2}\right)^{\mathrm{a}}$ & $25.7[23.8-27.8]$ & $30.2[27.6-33.6]$ & $<0.001$ \\
\hline Waist circumference $(\mathrm{cm})$ & $93.1(90.9,95.3)$ & $106.9(104.9,108.9)$ & $<0.001$ \\
\hline Systolic BP (mmHg) & $137(133,141)$ & $138(136,140)$ & 0.664 \\
\hline Diastolic BP (mmHg) & $75.6(73.8,77.4)$ & $76.2(74.8,77.5)$ & 0.654 \\
\hline MAP (mmHg) & $96.1(93.9,98.3)$ & $96.7(95.3,98.2)$ & 0.619 \\
\hline ABPI right & $1.15(1.12,1.18)$ & $1.15(1.12,1.17)$ & 0.83 \\
\hline ABPI left & $1.14(1.11,1.16)$ & $1.13(1.11,1.16)$ & 0.8 \\
\hline Total cholesterol $(\mathrm{mmol} / \mathrm{l})^{\mathrm{a}}$ & $4.80[4.10-5.70]$ & $3.90[3.30-4.50]$ & $<0.001$ \\
\hline LDL-cholesterol $(\mathrm{mmol} / \mathrm{l})^{\mathrm{a}}$ & $2.70[1.98-3.31]$ & $1.88[1.53-2.38]$ & $<0.001$ \\
\hline HDL-cholesterol (mmol/l) $)^{\mathrm{a}}$ & $1.53[1.23-1.92]$ & $1.24[1.06-1.43]$ & $<0.001$ \\
\hline $\mathrm{HbA}_{1 \mathrm{c}}(\mathrm{mmol} / \mathrm{mol})^{\mathrm{a}}$ & $40[38-42]$ & 57 [49-67] & $<0.001$ \\
\hline $\mathrm{HbA}_{1 \mathrm{c}}(\%)^{\mathrm{a}}$ & $5.7[5.5-6.0]$ & $7.4[6.6-8.3]$ & $<0.001$ \\
\hline History of CVD (\%) & 43.4 & 47.4 & 0.536 \\
\hline On antihypertensive (\%) & 40.4 & 79.2 & $<0.001$ \\
\hline$\beta$-blocker & 21.4 & 33.8 & 0.035 \\
\hline ACE-inhibitor & 20.4 & 51.3 & $<0.001$ \\
\hline Angiotensin receptor A & 6.1 & 17.5 & 0.009 \\
\hline On statin therapy (\%) & 45.9 & 81.8 & $<0.001$ \\
\hline \multicolumn{4}{|l|}{ Smoking status (\%) } \\
\hline Current & 5.1 & 4.6 & \\
\hline Previous & 48.0 & 50.7 & \\
\hline Never & 46.9 & 44.8 & 0.911 \\
\hline $\operatorname{AER}(\mu \mathrm{g} / \mathrm{min})^{\mathrm{a}}$ & $4.49[3.01-6.07]$ & $5.71[3.88-11.65]$ & $<0.001$ \\
\hline $\operatorname{ACR}(\mathrm{mg} / \mathrm{mmol})^{\mathrm{a}}$ & $0.72[0.53-1.10]$ & $1.07[0.59-2.17]$ & 0.014 \\
\hline \multicolumn{4}{|l|}{ Retinopathy $(n)$} \\
\hline No retinopathy & NA & 87 & NA \\
\hline Nonproliferative retinopathy & NA & 52 & NA \\
\hline Proliferative & NA & 0 & NA \\
\hline Laser treatment & NA & 4 & NA \\
\hline Unknown & NA & $11^{\mathrm{b}}$ & NA \\
\hline Neuropathy (\%) & NA & $24^{\mathrm{c}}$ & NA \\
\hline
\end{tabular}

Data are displayed as mean $(95 \% \mathrm{CI})$ or median IQR

${ }^{a}$ Skewed variable; median and IQR presented and $p$ for difference of the appropriately transformed data

${ }^{\mathrm{b}}$ Unknown retinopathy score owing to ungradable two-field photography or data not available on clinical database

${ }^{\mathrm{c}}$ Defined as neurothesiometer measurement $\geq 25 \mathrm{~V}$, available in 146 of 154 participants with type 2 diabetes ABPI, ankle brachial pressure index; ACR, albumin/creatinine ratio; F, female; M, male; MAP, mean arterial pressure; NA, not applicable almost twice as many with diabetes were receiving antihypertensive therapy. People with type 2 diabetes received a variety of glucose-lowering treatments: $20.1 \%$ were treated with diet only, $61.7 \%$ received oral glucose-lowering medication, $5.2 \%$ received insulin and $13.0 \%$ were treated with a combination of oral medication and insulin.
Data for change in metabolic and microvascular variables over 3 years are presented in Table 2. AER was higher at baseline and follow-up in those with diabetes compared with those without; however, this was below the range for clinically significant microalbuminuria (Table 2; difference between those with and those without 


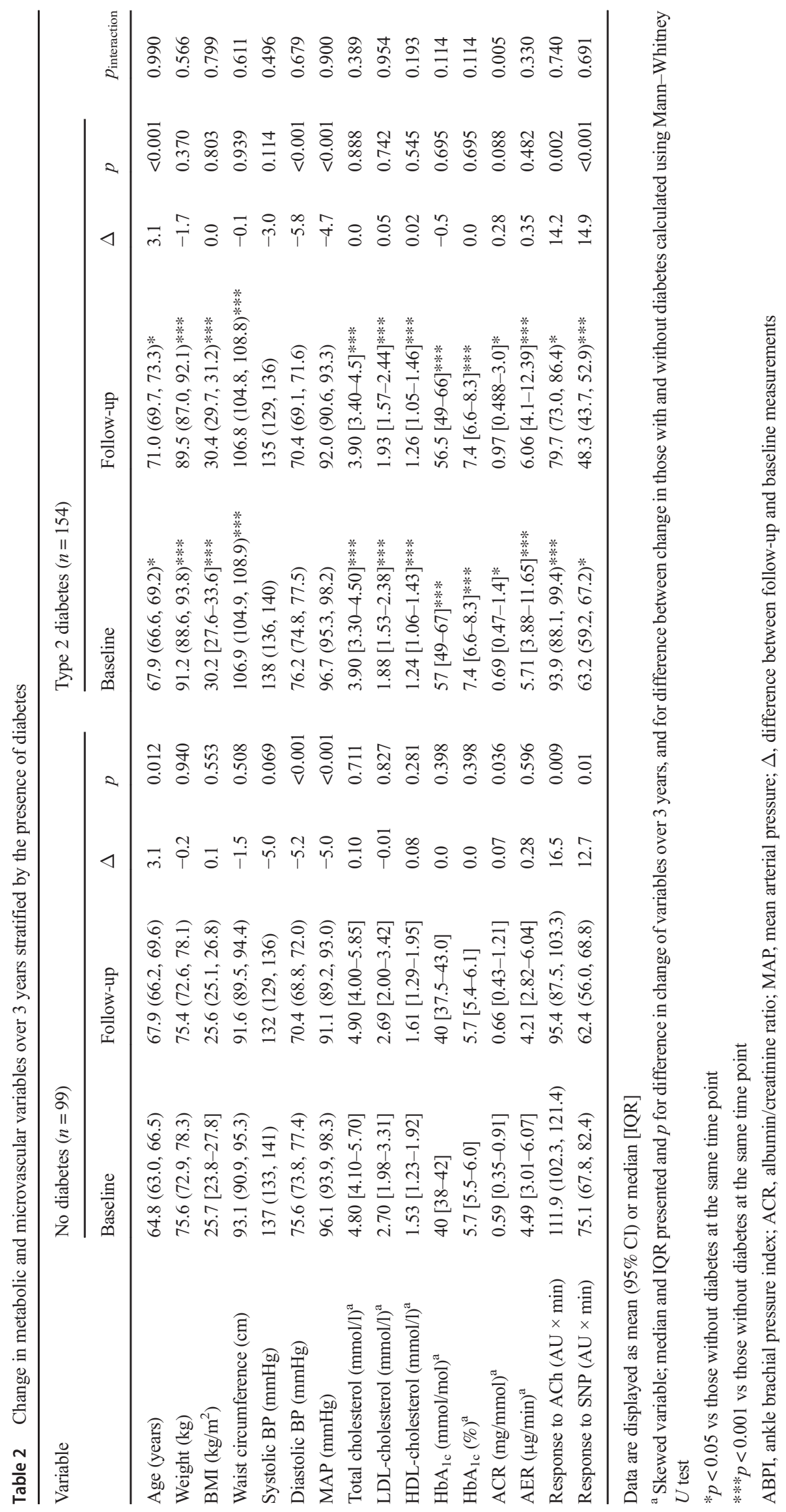


type 2 diabetes at both baseline and follow-up, $p<0.001$ ). Over 3 years, there was no significant increase in AER at this preclinical level, nor was there any difference in change over 3 years between those with and those without diabetes $\left(p_{\text {interaction }}=0.330\right)$.

At both baseline and follow-up, endothelium-dependent (ACh) and -independent (SNP) microvascular responses were attenuated in people with type 2 diabetes compared with those without (Table 2, Fig. 1a,b). Over 3 years, there was a similar decline in mean microvascular function in the groups with and without diabetes.
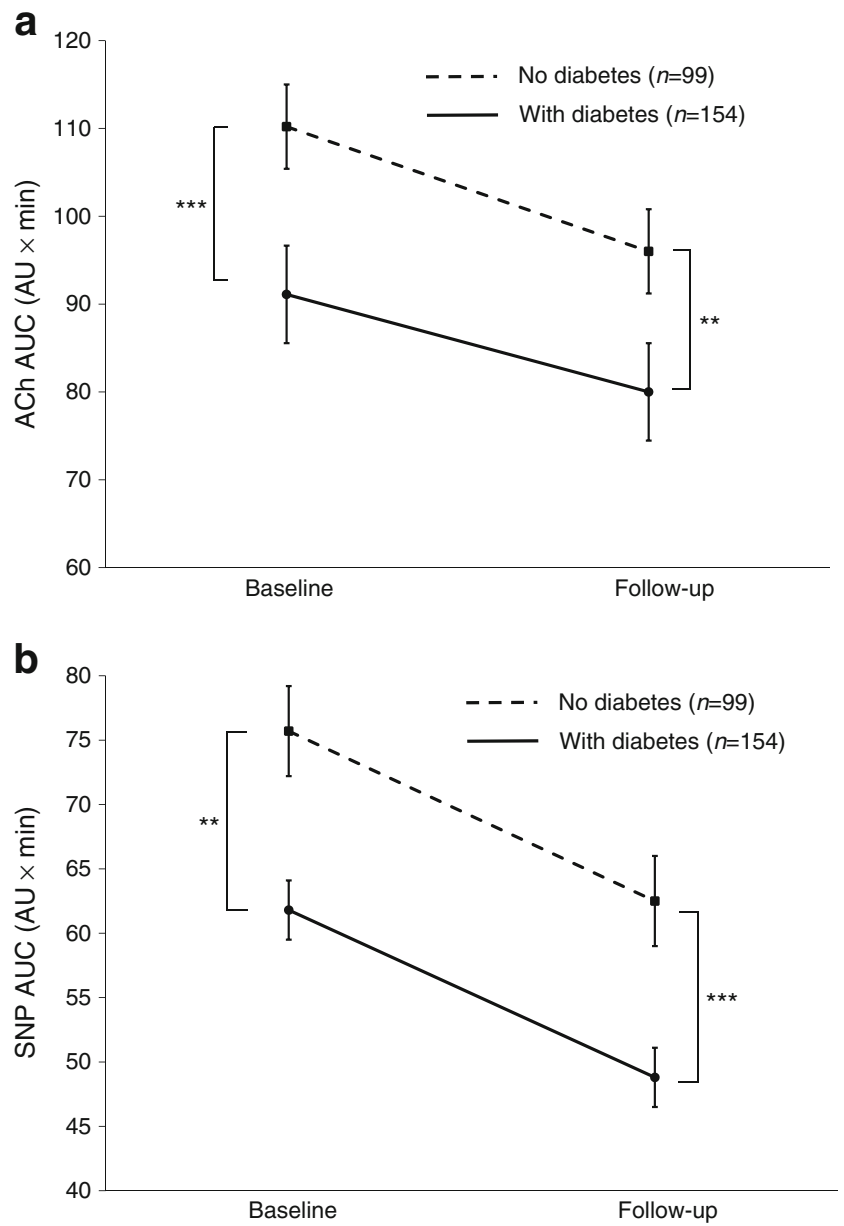

Fig. 1 Endothelial-dependent (a) and -independent (b) microvascular response at baseline and follow-up in participants with and without diabetes, over a 3 year period, measured as microvascular perfusion in response to Ach (a) and SNP (b). Data are presented as the mean AUC of the response curve $(\mathrm{AU} \times \min \pm \mathrm{SEM})$. Two-way ANOVA (grouped by time) was used to test interaction (a $p=0.740 ; \mathbf{b} p=0.691)$. (a) People with diabetes had attenuated microvascular function in response to ACh at baseline $(* * * p<0.001)$ and follow-up $(* * p<0.01)$. There was significant change in ACh over 3 years in people both with $(p=0.002)$ and without type 2 diabetes $(p=0.009)$. (b) People with diabetes had attenuated microvascular function in response to SNP at baseline $(* * p<0.01)$ and follow-up $(* * * p 0.001)$. There was significant reduction in SNP over 3 years in people both with $(p<0.001)$ and without type 2 diabetes $(p=0.01)$
Determinants of change in microvascular function over the 3 years Although the average decline in group mean microvascular function was similar in individuals both with and without diabetes (age- and sex-adjusted standardised $\beta=$ $0.016, p=0.8$; and $-0.05, p=0.4$ for change in ACh and $\mathrm{SNP}$, respectively), there was significant diversity of magnitude and direction of change within the populations. Exploring the whole cohort, percentage change in weight accounted for the greatest variance of the model, making it the single most predictive factor (Table 3 ) for the decline in microvascular function, such that a reduction in weight was associated with less of a deterioration of both endotheliumdependent and -independent microvascular function, whereas weight gain was associated with a greater decline in function. All parameters of change in body composition and adiposity, including absolute change in weight, change in BMI and change in waist/hip ratio, gave similar results. Those on statin therapy saw less of a decline in endothelium-dependent function compared with those not on statin therapy. There was no association between change in microvascular function and history of CVD, change in any blood pressure variable, any blood pressure intervention (including $\beta$-blockers or modifiers of the renin angiotensin aldosterone system), change in HDL-cholesterol or change in LDL-cholesterol.

In those with diabetes alone, change in weight was the strongest predictor of changes in endothelial-dependent and -independent microvascular function (Table 3). Numerically, this was greater by approximately a quarter for endothelial-dependent and $45 \%$ for endothelial-independent function than in those without diabetes, although this interaction was not statistically significant ( $p_{\text {interaction }}=0.6$ and 0.5 for ACh and SNP, respectively). Change in glycaemic control $\left(\mathrm{HbA}_{1 \mathrm{c}}\right)$ was also associated with a modest change in endothelial-dependent microvascular function over time, such that an increase in $\mathrm{HbA}_{1 \mathrm{c}}$ was associated with a poorer function independent of change in weight. Within a small cohort of participants, interim $\mathrm{HbA}_{1 \mathrm{c}}$ values were available. For these individuals, we stratified into those with 'good control', with a mean $\mathrm{HbA}_{1 \mathrm{c}}<53 \mathrm{mmol} / \mathrm{mol}(7.0 \%)$ over the 3 years, and those with less good control, with a mean $\mathrm{HbA}_{1 \mathrm{c}}>$ $64 \mathrm{mmol} / \mathrm{mol}$ (8.0\%; electronic supplementary material [ESM] Fig. 1). Those with good glycaemic control had less attenuation of microvascular function over the 3 years compared with those with poorer control $(p=0.03)$; however, this was accounted for by less weight gain in those with better glycaemic control ( $p$ after adjustment 0.2$)$. The use of sulfonylureas ( $n=46,29.9 \%$ of the population with diabetes) was associated with a greater decline in endothelial microvascular function compared with those with diabetes on other therapies, including insulin, after adjustment for the $\mathrm{HbA}_{1 \mathrm{c}}$ effect (adjusted standardised $\beta$ 0.199, $p=0.02$ ). This association was independent of change in weight, other diabetes therapies and duration of diabetes (adjusted standardised $\beta$ 0.198, $p=0.02$ ). An exploratory analysis determining the impact of going into remission from diabetes (defined as 
Table 3 Association of changes in microvascular function with conventional cardiovascular risk factors and treatment in the whole population and in those with and without diabetes after adjustment for age and sex

\begin{tabular}{|c|c|c|c|c|}
\hline \multirow[t]{2}{*}{ Variable } & \multicolumn{2}{|l|}{ Change in $\mathrm{ACh}$} & \multicolumn{2}{|l|}{ Change in SNP } \\
\hline & Standardised $\beta$ & $p$ value & Standardised $\beta$ & $\mathrm{p}$ value \\
\hline \multicolumn{5}{|l|}{ Whole cohort } \\
\hline History of CVD & 0.069 & 0.292 & -0.121 & 0.065 \\
\hline Percentage change in weight & -0.248 & $<0.001$ & -0.165 & 0.009 \\
\hline Change in diastolic blood pressure & -0.058 & 0.374 & -0.030 & 0.647 \\
\hline Change in LDL-cholesterol & 0.103 & 0.138 & 0.071 & 0.306 \\
\hline Statin treatment at baseline & 0.160 & 0.016 & -0.015 & 0.821 \\
\hline \multicolumn{5}{|l|}{ Diabetes-specific analysis $(n=154)^{\mathrm{a}}$} \\
\hline History of CVD & 0.020 & 0.808 & -0.133 & 0.112 \\
\hline Percentage change weight & -0.317 & $<0.001$ & -0.240 & 0.003 \\
\hline Change in diastolic blood pressure & -0.076 & 0.363 & -0.056 & 0.504 \\
\hline Change in LDL-cholesterol & 0.016 & 0.853 & 0.034 & 0.704 \\
\hline Percentage change in $\mathrm{HbA}_{1 \mathrm{c}}$ & -0.203 & 0.014 & 0.082 & 0.329 \\
\hline Sulfonylurea treatment ${ }^{\mathrm{b}}$ & -0.199 & 0.022 & -0.112 & 0.209 \\
\hline \multicolumn{5}{|l|}{ People without diabetes $(n=99)$} \\
\hline History of CVD & 0.183 & 0.084 & -0.095 & 0.370 \\
\hline Percentage change in weight & -0.160 & 0.117 & -0.031 & 0.764 \\
\hline Change in diastolic blood pressure & -0.037 & 0.726 & -0.002 & 0.987 \\
\hline Change in LDL-cholesterol & 0.235 & 0.033 & 0.109 & 0.313 \\
\hline Statin treatment at baseline & 0.190 & 0.080 & -0.137 & 0.209 \\
\hline
\end{tabular}

Standardised $\beta$ (i.e. SD change in variable of interest per SD change in contributing factor) from multivariate modelling between the microcirculatory variable of interest and the mechanistic/confounding factor after adjustment for age and sex. A negative standardised $\beta$ represents a greater decline in microvascular function per increase in variable (e.g. per SD increase in weight there will be $\sim 1 / 4$ of an SD greater decline in endothelialdependent microvascular function over 3 years)

${ }^{\text {a }}$ Statin therapy not considered for people living with diabetes due to co-linearity with $81 \%$ of individuals being treated

${ }^{\mathrm{b}}$ In a model with all glucose-lowering drugs available accounted for
$\mathrm{HbA}_{1 \mathrm{c}}<48 \mathrm{mmol} / \mathrm{mol}$ [6.5\%] on no glucose-lowering medications) suggested a numerical improvement in endothelial function compared with those that remained with diabetes (ESM Table 1). However, no formal statistical analysis was performed for this exploratory analysis due the small numbers.

Thresholds of benefit To further explore the association between weight change and change in microvascular function, we divided our cohort into participants who, over the 3 years of the study, had increased, decreased or stable weight, defined as $5 \%$ or less variation (loss or gain) in their weight. The $5 \%$ threshold in weight reduction has recently been shown to reduce risk of cardiovascular events in patients suffering with type 2 diabetes [21]. One hundred and ninety participants had a stable weight and 40 participants lost $5 \%$ or more of their weight (median $8 \%$ weight loss), including one participant who underwent bariatric surgery, whereas 22 participants gained $5 \%$ or more of their weight (median $7.3 \%$ weight gain). Endothelial-dependent microvascular response to ACh did not decline in those who lost $\geq 5 \%$ over the 3 years compared with those who had stable weight, whereas those who gained $\geq 5 \%$ weight had an exaggerated attenuation in microvascular function (change in endothelial-dependent function was 1.2 [95\% CI $-13.2,15.7] \mathrm{AU} \times \min$ in those who lost weight; $-15.8[-10.5,-21.0] \mathrm{AU} \times \min$ in those with stable weight; and $-37.8[-19.4,-56.2] \mathrm{AU} \times \min$ in those who put on $\geq 5 \%$ weight; Fig. 2). Adjustment for age, sex, changes in blood pressure, blood glucose and cholesterol did not change the association (ESM Table 2). These differences were numerically similar in those with and without diabetes, although, possibly due to the reduced power in the smaller number without diabetes, the significance was only maintained in those with diabetes ( $p=0.025$ for weight loss vs stable weight, $p<0.001$ for weight loss vs weight gain and $p=$ 0.007 for weight gain vs weight stable). The effect of weight loss in those with diabetes resulted in an endothelialdependent microvascular response after 3 years that was similar to those without diabetes who had stable weight over this time period (response in people with diabetes who lost weight $=101.8 \mathrm{AU} \times \min$ vs people without diabetes who maintained their weight 94.1 $\mathrm{AU} \times \min$ ). No formal testing was done on these comparisons. 


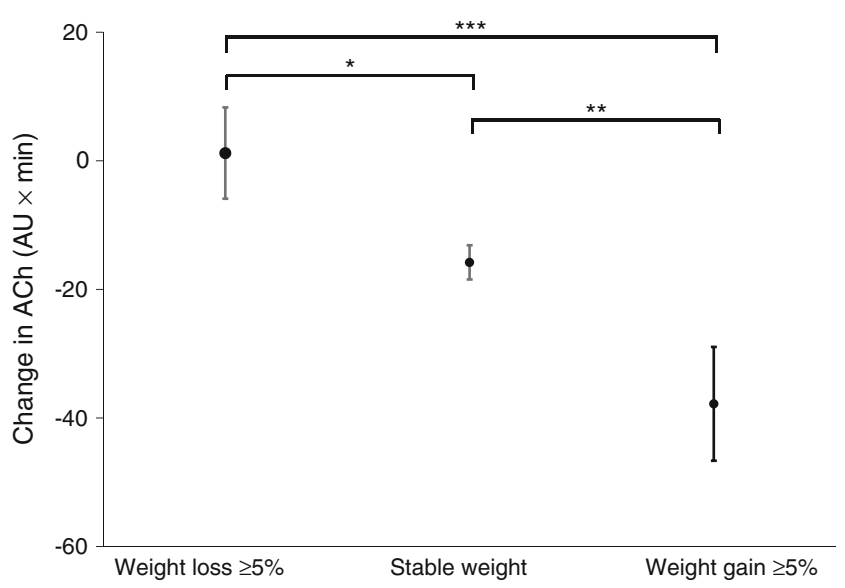

Fig. 2 Change in AUC in response to endothelial-dependent (ACh) stimulation over 3 years stratified into those that had $\geq 5 \%$ weight loss, those that were weight stable and those that gained $\geq 5 \%$ of their weight (AU $\times$ $\min \pm \mathrm{SEM}) . p$ values from $t$ tests, $* p<0.05, * * p<0.01$ and $* * * p<0.001$

For endothelial-independent response to SNP in the whole group, there was only a trend towards difference between those who lost $\geq 5 \%$ over the 3 years and those who had stable weight $(p=0.1)$. This trend appeared to be driven entirely by a significant difference in those with diabetes, such that those who lost $\geq 5 \%$ weight had a lower decline in SNP response compared with those who remained weight stable $(-4.5[4.6,-13.6]$ vs $-16.6[-12.0,-21.2] \mathrm{AU} \times \min ; p=0.02)$ and those who gained weight $(-4.5[4.6,-13.6]$ vs $-21.3[-9.2,-33.4] \mathrm{AU}$ $\times \min ; p=0.03$ ). There was no difference between those who had stable weight and those who gained weight $(p=0.43)$.

\section{Discussion}

We have demonstrated for the first time that, over a 3 year period, endothelial-dependent microvascular function declines in weight-stable individuals, but that this decline is attenuated with a modest amount of weight loss and accelerated with a modest amount of weight gain. This was found in a mixed cohort of people with and without type 2 diabetes. Importantly, our findings were independent of the measures of blood pressure and cholesterol we used and also independent of the treatment regimens. In individuals with type 2 diabetes, change in weight and change in $\mathrm{HbA}_{1 \mathrm{c}}$ were independent predictors of change in endothelial-dependent response, but only change in weight was associated with change in endothelial-independent response.

The attenuation of decline in ACh responses in the absence of an impact on SNP response in this study suggests that weight loss is associated with an improvement in endothelial function. This is in keeping with the growing understanding that obesity impairs endothelial function through systemic vascular inflammation [22].
Increased BMI and adiposity have an adverse effect on the microcirculation, independent of diabetes, in part due to the cellular inflammatory response to ischaemia occurring once the hypertrophy of adipocytes is beyond the $150 \mu \mathrm{m}$ diffusion range of oxygen [23]. Previous studies have shown that weight loss is associated with improvements in cyclical changes in microvascular perfusion (vasomotion) [24] and decreased proteinuria [25], but these studies involved significant weight loss interventions such as gastric banding and bypass surgery. Unlike these previous investigations, the 5\% weight loss that demonstrated benefit within our study is of modest magnitude and achievable without drastic interventions. In fact, we have shown that a weight reduction of at least $5 \%$ or more of body weight was associated with clear benefits in endothelial-dependent microvascular function.

A weight loss of at least $5 \%$ has been shown to have other beneficial effects in type 2 diabetes. Recently, for example, this weight change threshold has been shown to be associated with improvement in $\mathrm{HbA}_{1 \mathrm{c}}$ (a finding replicated here) and a lower 10 year hazard of CVD [21] in newly diagnosed type 2 diabetes patients. Our previous results [15] support the results of Strelitz et al [21], suggesting that the benefits of a $5 \%$ weight loss are wide ranging and that weight loss should be strongly encouraged.

This study has also shown that, in people living with diabetes, improving glycaemic control is associated with a lower decline in microvascular function over 3 years. It is important to consider whether this represents a mechanistic pathway, or simply confounding based on shared risk factors. However, this finding is supported by previous studies which demonstrated that tight glycaemic control reduced microvascular complications [26, 27]. Our study significantly adds to the current knowledge by demonstrating that these benefits can be realised at a stage prior to patients having microvascular complications, and therefore should be encouraged as soon as possible for people living with diabetes.

We found no difference in change in microvascular function over 3 years between people with and without diabetes. This was an unexpected finding; however, the association between direction of glycaemic control and microvascular function suggests that the absence of a difference may be due to population-wide improvement in glycaemic control in the group of individuals with diabetes, and a move away from sulfonylurea therapy over the 3 years. It is also important to note that, in the individuals with diabetes, lower microvascular function at baseline remained poorer at follow-up. The benefit of weight loss appeared to be exaggerated in those with diabetes compared with those without, although this difference did not reach statistical significance. There are several potential explanations for this. It may be that the lower baseline microvascular function in those with diabetes gives more potential for benefit of weight loss. There may be additional benefit in people with diabetes, given that insulin resistance is dependent on obesity, 
and thus weight loss improves the microvascular function directly and indirectly through improvement in the nonglycaemic elements of diabetes. Finally, it may simply be coincidental, given that the study was not powered to explore differences between those with and without diabetes but rather to assess the impact of diabetes itself.

Strengths and limitations To our knowledge, this is the largest study to have followed the natural history of microvascular function in people with and without diabetes over 3 years, who are treated in accordance with common practice within the UK. By evaluating a general population sample, with and without CVD, unlike the tight control that is delivered in randomised controlled trials, these results are representative of what a healthcare provider can reasonably expect to achieve working with their patient population. The observational nature, however, means it is impossible to ascribe causation. There also remains the potential for systematic bias, in terms of health-seeking behaviour and adherence to therapies prescribed. This is more likely to be greater in populations that also adhere to lifestyle suggestions. As a result, weight loss may simply be a proxy for medication adherence. Additionally, the study cannot exclude shared confounding of weight loss and improvement in microvascular endothelial function from other, unmeasured mechanistic factors, although the failure of conventional risk factors to account for these associations, having only a minimal effect on the standardised $\beta$ s, makes this unlikely. Further, as a secondary analysis of a subpopulation from the SUMMIT cohort, study power was limited for subgroup analyses. Across the whole population with the complete microvascular dataset, we have $97 \%$ power to detect a clinically meaningful $7 \%$ variance $\left(R^{2}\right)$ that was accounted for by difference in weight. Within the diabetes subpopulation, however, this power is reduced to $84 \%$, and further reduced to $64 \%$ for those without diabetes. This likely accounts for the lack of significant benefit demonstrated in the population without diabetes, despite similar numerical benefit as in those with diabetes.

Conclusion In conclusion, we have demonstrated that modest weight loss and good glycaemic control are associated with less 3 year decline in microvascular function in people with diabetes. Unlike studies in laboratory settings, where experimental conditions are tightly controlled, we observed the microvascular progression in a large group of people managed in the community by their usual physicians and no additional study-specific interventions. Further, we have demonstrated that a modest weight loss of only $5 \%$ attenuated the timedependent decline of endothelial-dependent microvascular function over 3 years.

Acknowledgements This work was supported by the Innovative Medicines Initiative (the SUMMIT consortium, IMI-2008/115006).
This paper presents independent research supported by the NIHR Exeter Clinical Research Facility and the NIHR Collaboration for Leadership in Applied Health Research and Care (CLAHRC) for the South West Peninsula. The views expressed in this publication are those of the author(s) and not necessarily those of the NIHR Exeter Clinical Research Facility, the NHS, the NIHR or the Department of Health in England. We would also like to acknowledge and thank the South West Stroke Research Network and the South West Retinopathy Service for their help with patient recruitment, and all of the patients who took part in this research. Some of the data were presented as an abstract at the 69th annual meeting of the British Microcirculation Society in 2019.

Data availability Original data are available by direct application to the corresponding author.

Funding This work was supported by the Innovative Medicines Initiative (the SUMMIT consortium, IMI-2008/115006).

Authors' relationships and activities The authors declare that there are no relationships or activities that might bias, or be perceived to bias, their work.

Contribution statement ACS, KMG, PEG and WDS were involved in study design and securing funding. FC, DDA, DM, CB, CA and KA were involved in study management and data collection. All authors have contributed to database analysis. FC, ACS and WDS performed the statistical analysis and produced the primary manuscript. All authors have seen and contributed to the drafting and revision of the manuscript and have approved the final version for publication. WDS is responsible for the integrity of the work as a whole.

Open Access This article is licensed under a Creative Commons Attribution 4.0 International License, which permits use, sharing, adaptation, distribution and reproduction in any medium or format, as long as you give appropriate credit to the original author(s) and the source, provide a link to the Creative Commons licence, and indicate if changes were made. The images or other third party material in this article are included in the article's Creative Commons licence, unless indicated otherwise in a credit line to the material. If material is not included in the article's Creative Commons licence and your intended use is not permitted by statutory regulation or exceeds the permitted use, you will need to obtain permission directly from the copyright holder. To view a copy of this licence, visit http://creativecommons.org/licenses/by/4.0/.

\section{References}

1. Strain WD, Cos X, Hirst M et al (2014) Time to do more: addressing clinical inertia in the management of type 2 diabetes mellitus. Diabetes Res Clin Pract 105(3):302-312. https://doi.org/10.1016/j. diabres.2014.05.005

2. Sedding DG, Boyle EC, Demandt JAF et al (2018) Vasa vasorum angiogenesis: key player in the initiation and progression of atherosclerosis and potential target for the treatment of cardiovascular disease. Front Immunol 9:706. https://doi.org/10.3389/fimmu. 2018.00706

3. Chantler PD, Frisbee JC (2015) Arterial function in cardiometabolic diseases: from the microcirculation to the large conduits. Prog Cardiovasc Dis 57(5):489-496. https://doi.org/10.1016/j. pcad.2014.09.005

4. Rizzoni D, Agabiti Rosei E (2006) Small artery remodeling in hypertension and diabetes. Curr Hypertens Rep 8(1):90-95. https://doi.org/10.1007/s11906-006-0046-3 
5. Laurent S, Boutouyrie P (2015) The structural factor of hypertension: large and small artery alterations. Circ Res 116(6):1007-1021. https://doi.org/10.1161/CIRCRESAHA.116.303596

6. Schofield I, Malik R, Izzard A, Austin C, Heagerty A (2002) Vascular structural and functional changes in type 2 diabetes mellitus: evidence for the roles of abnormal myogenic responsiveness and dyslipidemia. Circulation 106(24):3037-3043. https://doi. org/10.1161/01.CIR.0000041432.80615.A5

7. Casanova F, Adingupu DD, Adams F et al (2017) The impact of cardiovascular co-morbidities and duration of diabetes on the association between microvascular function and glycaemic control. Cardiovasc Diabetol 16(1):114. https://doi.org/10.1186/s12933017-0594-7

8. Nguyen TT, Kawasaki R, Wang JJ et al (2009) Flicker light-induced retinal vasodilation in diabetes and diabetic retinopathy. Diabetes Care 32(11):2075-2080. https://doi.org/10.2337/dc09-0075

9. Strain WD, Adingupu DD, Shore AC (2012) Microcirculation on a large scale: techniques, tactics and relevance of studying the microcirculation in larger population samples. Microcirculation 19(1): 37-46. https://doi.org/10.1111/j.1549-8719.2011.00140.x

10. Hansen PR, Haunso S (1995) Effects of nitric oxide synthase inhibition on myocardial capillary permeability and reactive hyperaemic response. Cardiovasc Res 29(6):862-866

11. Bottcher M, Madsen MM, Refsgaard J et al (2001) Peripheral flow response to transient arterial forearm occlusion does not reflect myocardial perfusion reserve. Circulation 103(8):1109-1114. https://doi.org/10.1161/01.cir.103.8.1109

12. Branten AJ, Smits P, Jansen TL, Wollersheim H, Thien T (1996) Effect of atrial natriuretic factor on skin microcirculation versus skeletal muscle blood flow. J Cardiovasc Pharmacol 27(2):303306. https://doi.org/10.1097/00005344-199602000-00019

13. Morris SJ, Shore AC, Tooke JE (1995) Responses of the skin microcirculation to acetylcholine and sodium nitroprusside in patients with NIDDM. Diabetologia 38(11):1337-1344. https:// doi.org/10.1007/bf00401767

14. Turner J, Belch JJ, Khan F (2008) Current concepts in assessment of microvascular endothelial function using laser Doppler imaging and iontophoresis. Trends Cardiovasc Med 18(4):109-116. https:// doi.org/10.1016/j.tcm.2008.02.001

15. Strain WD, Chaturvedi N, Hughes A et al (2010) Associations between cardiac target organ damage and microvascular dysfunction: the role of blood pressure. J Hypertens 28(5):952-958. https:// doi.org/10.1097/HJH.0b013e328336ad6c

16. Strain WD, Chaturvedi N, Bulpitt CJ, Rajkumar C, Shore AC (2005) Albumin excretion rate and cardiovascular risk: could the association be explained by early microvascular dysfunction? Diabetes 54(6):1816-1822. https://doi.org/10.2337/diabetes.54.6. 1816
17. Nguyen TT, Shaw JE, Robinson C et al (2011) Diabetic retinopathy is related to both endothelium-dependent and -independent responses of skin microvascular flow. Diabetes Care 34(6):1389 1393. https://doi.org/10.2337/dc10-1985

18. Khan F, Patterson D, Belch JJ, Hirata K, Lang CC (2008) Relationship between peripheral and coronary function using laser Doppler imaging and transthoracic echocardiography. Clin Sci (Lond) 115(9):295-300. https://doi.org/10.1042/CS20070431

19. Strain WD, Hughes AD, Mayet J et al (2013) Attenuated systemic microvascular function in men with coronary artery disease is associated with angina but not explained by atherosclerosis. Microcirculation 20(7):670-677. https://doi.org/10.1111/micc. 12066

20. Loader J, Khouri C, Taylor F et al (2019) The continuums of impairment in vascular reactivity across the spectrum of cardiometabolic health: a systematic review and network meta-analysis. Obes Rev 20(6):906-920. https://doi.org/10.1111/obr.12831

21. Strelitz J, Ahern AL, Long GH et al (2019) Moderate weight change following diabetes diagnosis and 10 year incidence of cardiovascular disease and mortality. Diabetologia 62(8):13911402. https://doi.org/10.1007/s00125-019-4886-1

22. Engin A (2017) Endothelial dysfunction in obesity. Adv Exp Med Biol 960:345-379. https://doi.org/10.1007/978-3-319-48382-5_15

23. Sorop O, Olver TD, van de Wouw J et al (2017) The microcirculation: a key player in obesity-associated cardiovascular disease. Cardiovasc Res 113(9):1035-1045. https://doi.org/10.1093/cvr/ cvx093

24. Rossi M, Nannipieri M, Anselmino M et al (2011) Skin vasodilator function and vasomotion in patients with morbid obesity: effects of gastric bypass surgery. Obes Surg 21(1):87-94. https://doi.org/10. 1007/s11695-010-0286-9

25. Afshinnia F, Wilt TJ, Duval S, Esmaeili A, Ibrahim HN (2010) Weight loss and proteinuria: systematic review of clinical trials and comparative cohorts. Nephrol Dial Transplant 25(4):11731183. https://doi.org/10.1093/ndt/gfp640

26. Action to Control Cardiovascular Risk in Diabetes Study G, Gerstein HC, Miller ME et al (2008) Effects of intensive glucose lowering in type 2 diabetes. N Engl J Med 358(24):2545-2559. https://doi.org/10.1056/NEJMoa0802743

27. Stratton IM, Adler AI, Neil HA et al (2000) Association of glycaemia with macrovascular and microvascular complications of type 2 diabetes (UKPDS 35): prospective observational study. BMJ 321(7258):405-412. https://doi.org/10.1136/bmj.321.7258. 405

Publisher's note Springer Nature remains neutral with regard to jurisdictional claims in published maps and institutional affiliations. 